\title{
Functional skills after the rehabilitation of spinal cord injury patients: observation period of 3 years
}

I Dzidic and S Moslavac

Spinal Center of the Specialised Hospital for Medical Rehabilitation, 42223 Varazdinske Toplice, Croatia

\begin{abstract}
The increased number of young people with spinal cord injuries in Croatia led us to study their functional abilities after completing medical rehabilitation and extended hospitalisation, as due to destroyed homes, they had no place to return to. They were all wounded during war in Croatia (1990-1992) and their average age was 27 years. Our tetraplegic patients achieved a mean Modified Barthel Index (MBI) of 57.3 after completing medical rehabilitation and 61.3, 3 years later, which is a significantly higher rate than is given in Yarkony's study (30.337.8) where American civilian patients were monitored. ${ }^{1}$ Patients with paraplegia showed similar results $(74.5-76.8)$ relating to those published by Yarkony $(72.0-75.6)$.
\end{abstract}

Keywords: spinal cord injury, tetraplegia; paraplegia; rehabilitation of spinal cord injuries; Croatia

\section{Introduction}

During the last decades it became apparent that patients with spinal cord injuries retain or even improve their functional abilities after completing primary rehabilitation. Thus Berns et al published in $1967^{2}$ reported that 31 patients with spinal cord injury retained their attained functional abilities at least for 1 year after leaving hospital. Grynbaum and coworkers ${ }^{3}$, however, state that certain functional skills, such as transfer and propelling a wheelchair, often deteriorate after discharge from hospital because of inadequate housing conditions. Scranton et $a l^{4}$ Rogers and Figone, ${ }^{5}$ as well as Runge, ${ }^{6}$ found that there was no change of functional abilities after discharge. NorrisBaker's group ${ }^{7}$ noticed that patients who were engaged in numerous activities, after discharge from hospital had a better chance for life on their own. According to their data, patients who were mobile in the hospital integrate better into the activities of their social surrounding.

However, all of these studies, even though useful regarding information relating to subsequent functional abilities of patients with spinal cord injuries, were often limited. The reasons being that these were very small test groups, inadequate functional provisions, no continuous observation period, and also a lack of statistical analysis. In 1988, Yarkony et al published a study of 236 patients with spinal cord injuries, whom they observed during 3 years after rehabilitation, using the Modified Barthel Index $(\mathrm{MBI})^{8-10}$ to rate their functional abilities. Their findings will be presented in comparison with ours subsequently in this article.

Correspondence: S Moslavac
We tried to define the functional skills of our SCI patients who, unlike others so far described in the literature, for various reasons remained in our hospital for even more than 3 years.

During the aggression almost a third of Croatia's housing facilities were destroyed or damaged and a great part of the Croatian territory was occupied. Unfortunately, this also happened to most of our patients' homes and, despite our wish for their resocialization after completed medical rehabilitation, they had no place to return to. To enable them to have suitable care and organization, they remained in our hospital. Following the liberation of occupied territories and with the start of reconstruction of houses and other facilities devastated in war, our spinal patients will leave hospital and will try to reorganize their communial life.

We compared our data with those from the Rehabilitation Institute of Chicago, published by Yarkony et $a l^{1}$ in an attempt to evaluate, by means of the well recognized Modified Barthel Index, the functional skills of our patients and thus indirectly discover the results of our efforts in their rehabilitation. We also wanted to examine whether such long hospitalization affects functional outcomes, referring to Yoshida's study. ${ }^{11}$ We assumed that very long hospitalisation could result in a worsening of functional abilities of those young people.

\section{Methods}

Initial rehabilitation in our Spinal Center used to begin after the stabilization of the patients' general condition, which is usually the 14th postoperative day for 
paraplegic and paraparetic patients', and after the stabilization of respiratory function for tetraplegic and tetraparetic individuals. A comprehensive individual program based on a multidisciplinary team approach is carried out. Members of the team are physiatrists, physical, occupational and recreational therapists, nurses, psychologists, social workers and other experts (neurosurgeons, orthopedists, neurologists, psychiatrists, urologists and internal medicine specialists) with the help provided by colleagues from WHO (World Health Organization).

Kinesitherapy, hydrotherapy, electrotherapy, psychotherapy and vocational rehabilitation is provided. Special care is achieved in urinary tract evaluation (standard and urodynamic studies) and a bladder and bowel program. Within a period of 3-6 months after rehabilitation start, all patients were rehabilitated to the maximal state of independence possible at that time. Unfortunately, because of the already mentioned reasons, they could not, unlike American patients, leave hospital and go home. Instead they moved to another part of our Center where they spent their time more independently. During that prolonged period of hospitalization, kinesitherapy for the prevention of limitation of joint movement, and to maintain a good range of movement and also to reduce muscle spasms continued. We also try to involve our patients in sport activities as well as in our computer workshop. It is important to mention that our Spinal Center is the only one in Croatia.

We chose 30 patients with traumatic spinal cord injuries, who were, after their primary medical treatment and stabilization of their general condition, sent to our Spinal Center for rehabilitation, where they continued to be hospitalized. The observation refers to their stay from February 1991 until 1995. Within a period of 3-6 months after the onset of rehabilitation, all patients were rehabilitated to the maximal state of independence possible at that time. The average age was 27 years and all were males. 63\% (19) were paraplegic; $10 \%$ (3) paraparetic, $23 \%$ (7) tetraplegic and 3\% (1) tetraparetic. All injuries resulted from the war. The spinal cord injury level was defined as the most caudal spinal segment with normal neurological function. A complete lesion (plegia) was defined as one with no sensory or motor function below the level of injury. An incomplete lesion (paresis) was defined as one with any sensory or motor function below the level of spinal cord trauma.

The Modified Barthel Index ${ }^{8-10}$ is a scale of 100 points for the ability of performing different skills relating to personal care and mobility, and was estimated at the end of the rehabilitation period, as after 30-48 months later. The MBI scale proved to be very precise and reliable for recording functions and changes of the respective abilities at patients with a SCI. The special skills referred to were personal hygiene, taking a bath, food supply, transfer to bed and toilet, moving upstairs, dressing, bowel motion and urine control, as well as versatility with a wheelchair. We compensated the original drive to the subway with estimating the drive on a moving staircase in a department store.

Chi square analysis was used to compare attained mean MBI values in our and American SCI patient populations. ${ }^{1}$

\section{Results}

Table 1 presents patients according to diagnosis. Number of patients with each diagnosis, mean MBI values after completed medical rehabilitation (3-6 months following hospitalization and rehabilitation start) (MBI I) and 3 years later (MBI II) as well as mean improvement (MI) levels are listed. The findings of Yarkony et $a l^{1}$ are given for comparison. At the end of medical rehabilitation patients with paraplegia had mean MBI scores of 74.5, and with the continuance of stay 76.8. Patients with paraparesis finished their initial rehabilitation with mean MBI scores of 93.5, effecting later on 95.0. Those with tetraplegia showed mean MBI scores of 57.3 at the end of rehabilitation, respectively 61.3 after several years. The rate of the patient with tetraparesis improved within the same time from 80 to 91 .

All groups of patients improved functional independence levels throughout a 3 year period (Figure 1).

Using Chi square analysis we did not find a significant difference $(P<0.05)$ between our and American monitored paraplegic $(3.84>0.9865)$ and paraparetic $(3.84>0.9788)$ patients. However, in

Table 1 Functional skills measured by means of the Modified Barthel Index (MBI) at the end of medical rehabilitation (MBI I), 3 years later (MBI II), and mean improvement levels (MI) are listed. For comparison data are presented from Rehabilitation Institute of Chicago ${ }^{1}$

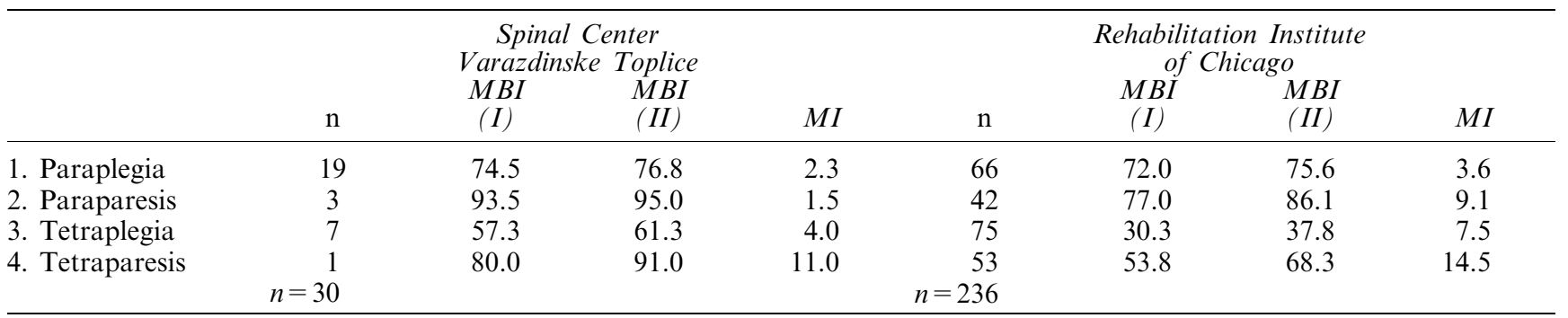




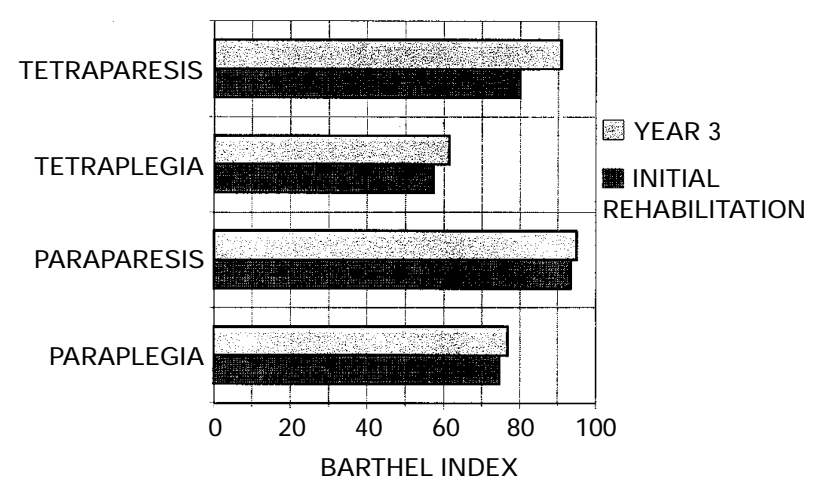

Figure 1 Mean Modified Barthel Index (MBI) scores for spinal cord injured patients at the end of medical rehabilitation and three years later

tetraplegic patients we observed significant difference $(P<0.05)$ between these two groups $(3.84<9.8571)$.

\section{Discussion}

Regarding the results published in the literature so far, ${ }^{11}$ our findings do not confirm a drop of functional abilities due to a very long period of hospitalization. Our results are in accord with data published in the literature. $^{1,2,7}$ An increase of the Modified Barthel Index, means an improvement of functional skills and could be proved for all categories of patients throughout several years. It appears that patients with a lower level of injury (paraplegia and paraparesis) show a higher mean MBI, respectively higher functional skills, than patients with higher level of injury (tetraplegia and tetraparesis).

The mean values of Modified Barthel Index of those with paresis are much higher than those with a complete injury-plegia. The best improvement after a period of $30-48$ months can be noticed in patient with tetraparesis (11.0) followed by patients with tetraplegia (4.0), paraplegia (2.3) and paraparesis (1.5) (see Table $1)$.

In their study Yarkony et $a l^{1}$ state a mean improvement of 7.5 for those with tetraplegia and 3.6 for those who were paraplegic. Our findings, however, show a somewhat lower mean improvement level, although the age of the patients must be taken into consideration. Thus, all of our patients were wounded when they were young and most were soldiers. Their health before injury, taking into account the army training before the trauma, influenced the motivation for a fast functional recovery. Therefore, we consider that our patients have achieved their best possible results already during their primary rehabilitation, so that subsequent improvements differ individually.

We observed significantly higher functional abilities/ MBI values at our patients with tetraplegia (57.361.3), than those published in Yarkony's study (30.337.8). ${ }^{1}$ Such favourable results can, additionally to the above mentioned reasons, be ascribed to the acute care that was given, based on urgent transport (mostly by helicopters) as well as the surgical treatment provided in the specialized Center of the Clinic for Traumatology at Zagreb, and furthermore to the appropriate rehabilitation treatment at the Spinal Center of Specialized Hospital Varazdinske Toplice. The excellent values of the Modified Barthel Index (80-91) and the resulting improvement of tetraparetic patients cannot be valued in a general way. Finally, our patients with paraplegia, who are the largest group in our study, have in both observed periods reached similar (74.5-76.8) effects, and these correspond to those published by Yarkony $(72.0-75.6){ }^{1}$

Comparing our findings measured by the MBI scale to those described in the literature, ${ }^{1}$ we found numerous similarities. A confirmation by observing more patients during an extended time period would surely be very valuable.

This study is particularly interesting as the patients remained hospitalized even after their medical rehabilitation had been completed. Despite a possible specific slackening and a negative influence of too long hospitalization on the psychological status of severely disabled patients, no reduction of functional skills and activities in the workaday routine could be noticed.

Therefore, we could not prove a decrease of functional abilities in our patients, even although we further consider that continued hospitalization after an achieved maximal independence as unfavourable. It is our opinion that an appropriate vocational orientation after completing medical rehabilitation, as well as adequate employment are the only correct solutions for the future life of these young people within a wider social environment.

\section{References}

1 Yarkony GM et al. Functional skills after spinal cord injury rehabilitation: three year longitudinal follow-up. Arch Phys Med Rehabil 1988; 69: 111-114.

2 Berns SH, Lowman EW, Rusk HA, Covalt DA. Spinal cord injury - rehabilitation costs and results and follow-up in thirtyone cases. JAMA 1957; 164: 1551-1558.

3 Grynbaum BB, Kaplan LI, Lloyd KE, Rusk HA. Methodology and initial findings in follow-up study of spinal cord dysfunction. Arch Phys Med Rehabil 1963; 44: 208 - 215.

4 Scranton J, Fogel ML, Erdman WJII. Evaluation of functional levels of patients during and following rehabilitation. Arch Phys Med Rehabil 1970; 51: $1-21$

5 Rogers JC, Figone JJ. Traumatic quadriplegia: follow-up study of self care skills. Arch Phys Med Rehabil 1980; 61: 316-321.

6 Runge M. Follow-up study of self-care activities in traumatic spinal cord injury quadriplegics and quadriparetics. Am J Occup Ther 1966; 20: 241 - 249.

7 Norris-Baker C, Stephens MAP, Rintala DH, Willems EP. Patient behavior as predictor of outcomes in spinal cord injury. Arch Phys Med Rehabil 1981; 62: $602-608$.

8 Granger CV, Albrecht GL, Hamilton BB. Outcome of comprehensive medical rehabilitation: measurement by PULSES profile and the Barthel Index. Arch Phys Med Rehabil 1979; 60: $145-154$. 
9 Mahoney FI, Barthel DW. Functional evaluation: Barthel index. Md State Med J 1965; 14: $61-65$.

10 Yarkony GM et al. Benefits of rehabilitation for traumatic spinal cord injury: multivariate analysis in 711 patients. Arch Neurol 1987; 44: $93-96$.
11 Yoshida KK. Institutional impact on self concept among persons with spinal cord injury. International Journal of Rehabilitation Research 1994; 17: 95-107. 